\title{
Classificação de Falhas em Motor de Indução Utilizando Curvas Principais
}

\author{
Fernando E. M. Borges*. Letycia M. Borges**, Diogo A. Ribeiro***. Andrey W. M. Pinto****. Danton D. \\ Ferreira***** \\ *Departamento de Automática, Universidade Federal de Lavras; Lavras, MG, Brasil \\ (e-mail:fborges@estudante.ufla.br). \\ **Departamento de Engenharia, Universidade Federal de Lavras; Lavras, MG, Brasil \\ (e-mail: letycia.borges@estudante.ufla.br). \\ ***Departamento de Automática, Universidade Federal de Lavras, Lavras, MG, Brasil \\ (e-mail: diogoaranhavga@gmail.com). \\ ****Departamento de Automática, Universidade Federal de Lavras, Lavras, MG, Brasil \\ (e-mail: andreymarques96@gmail.com). \\ *****Departamento de Automática, Universidade Federal de Lavras, Lavras, MG, Brasil (e-mail: danton@ufla.br).
}

\begin{abstract}
Electric motors are very important machines in any industrial plant, due to your large range of uses and robustness. Evaluate the conditions of this machines is crucial to ensure the operation with security and quality. This paper presents a methodology of fault classification using vibration analysis, the vibration signals were measured by a 3-axis accelerometer linked to un Arduino microcontroller. The front bearing was evaluated in good conditions and in two failures situations in its races. The feature extraction was realized by Higher-Order Statistics using cumulants of 2nd and 4th orders with zero lag and the classification was made using Principal Curves. Principal Curves are obtained to each motor condition and realized the classification by measure of the distances of each event to the curve. Classification results were obtained with hits rates above $95 \%$.
\end{abstract}

Resumo: Motores elétricos são máquinas de suma importância em qualquer planta industrial devido sua gama de aplicações e robustez. Avaliar as condições de tais máquinas é crucial para garantir que estes operem com segurança e qualidade. O presente artigo apresenta uma metodologia de classificação de falhas utilizando análise de vibrações, os sinais de vibração foram medidos por um acelerômetro de 3 eixos ligado a um microcontrolador Arduino. Foi avaliado o rolamento dianteiro do motor em boas condições e em duas situações de falhas nas pistas. A extração de parâmetros foi realizada por meio de Estatísticas de Ordem Superior, utilizando cumulantes de segunda e quarta ordens com atraso nulo e a classificação feita utilizando Curvas Principais. Foram obtidas Curvas Principais para cada condição do motor e realizada a classificação pela medida das distâncias de cada evento à curva. Resultados de classificação foram obtidos com valores de taxas de acerto acima de $95 \%$.

Keywords: Induction Motor; Principal Curves; Higher-Order Statistics; Vibration Analysis; Fault Classification.

Palavras-chaves: Motor de Indução; Curvas Principais; Estatísticas de Ordem Superior; Análise de Vibrações; Classificação de Falhas.

\section{INTRODUÇÃO}

Máquinas elétricas são utilizadas em qualquer planta industrial para as mais diversas aplicações existentes. Dentre tais máquinas, os motores de indução apresentam vasto uso devido à sua robustez, construção simples, além de poder ser utilizado nas mais diversas aplicações. Com a evolução da tecnologia, tais máquinas operam em condições cuja exigência é cada vez maior. Para atender à tais demandas, garantindo maior produtividade e segurança, novas tecnologias são estudadas e implementadas, como a conectividade e monitoramento de tais equipamentos. Tal tecnologia é conhecida como indústria 4.0 (Thoben et. al., 2017).
Diante deste cenário, a manutenção tende a migrar da forma de apenas corrigir defeitos, passando a analisar e monitorar as condições de máquinas e equipamentos, objetivando otimizar a vida útil dos componentes destas e programar paradas para a troca dos mesmos. Tal técnica é denominada manutenção preditiva ou manutenção baseada na condição (Liao e Wang, 2013).

No contexto de motores de indução, os rolamentos são os componentes com maior incidência de falhas. De acordo com Gongora et. al. (2016), a incidência de falhas em rolamentos chega a $44 \%$ das falhas em motores.

$\mathrm{Na}$ literatura, encontram-se trabalhos desenvolvidos para detecção de falhas em rolamentos utilizando diferentes 
abordagens. Tian et. al. (2016) utilizaram extração de características de sinais de vibração por meio de curtose espectral (SK) e classificação por meio do método Kvizinhos mais próximos para detecção de falhas em rolamentos em estágio incipiente. Yu et. al. (2013) propuseram, para detecção de falhas em rolamentos de um metrô, o uso de rede neural de função de base radial (RBF) aliado a um pré-processamento dos sinais usando denoising por meio de wavelets. Também utilizando pré-processamento por denoising, Darong et. al. (2018) utilizaram mínimos quadrados recursivos (RLS) combinados com decomposição por média local (LMD) para detecção de falhas em rolamentos.

Outros tipos de detecção de falhas também são estudados por meio de análises em frequência. Culbert e Letal (2017) propuseram um método baseado na análise em frequência dos sinais de corrente provenientes do motor para detecção de falhas no rotor e de desbalanceamento do vão entre o estator e o rotor. Park et. al. (2017) propuseram, além de uma análise para detecção de falhas por resposta em frequência, uma análise da contribuição da frequência das pás em uma bomba centrífuga para geração de falsos positivos. Os autores propuseram formas de detecção de falhas e discriminação das frequências das pás.

Neste artigo é proposto um método de classificação de padrões em um rolamento de um motor de indução. O diferencial do trabalho consiste no uso de Curvas Principais (CP) como classificador. As Curvas Principais são uma generalização não-linear da análise de componentes principais (PCA), são não paramétricas e sua forma é sugerida pelos dados que a projetam. Método proposto inicialmente por Hastie e Stuetzle (1989), possui diversas alternativas para obtenção de uma $\mathrm{CP}$, dentre as tais, o método k-segmentos, proposto por Verbeek, Vlassis e Krose (2001), utilizado neste trabalho. As CP possuem a capacidade de extrair modelos compactos dos dados, representando um conjunto de dados n-dimensionais em um modelo unidimensional, resultando assim, na alimentação de modelos de classificação simples e eficientes.

\section{MÉTODO PROPOSTO}

O método proposto baseia-se em 4 estágios: aquisição de sinais de vibração, extração de parâmetros, projeto do classificador e avaliação dos resultados. Tais estágios serão discutidos nas subseções seguintes e estão ilustrados na Fig. 1.

\begin{tabular}{|c|c|c|c|}
\hline $\begin{array}{l}\text { Aquisição de } \\
\text { Sinais }\end{array}$ & $\begin{array}{l}\text { Extração de } \\
\text { Parâmetros }\end{array}$ & $\begin{array}{l}\text { Projeto do } \\
\text { Classificador }\end{array}$ & $\begin{array}{l}\text { Análise dos } \\
\text { Resultados }\end{array}$ \\
\hline
\end{tabular}

Fig. 1 Fluxograma do método proposto.

\subsection{Aquisição de Sinais}

Os sinais de vibração foram coletados de um motor de indução marca KOHLBACH® de $2 \mathrm{CV}$, tensão de alimentação $380 \mathrm{~V}$ e rotação nominal de $3.480 \mathrm{rpm}$. O motor foi acionado por partida direta e operado a vazio. As falhas foram induzidas no rolamento dianteiro do motor de modelo NSK 6204. Dois tipos de falha foram introduzidos: um furo na pista externa do rolamento e outro nas duas pistas do rolamento.

A coleta dos sinais de vibração foi realizada mediante um acelerômetro de 3 eixos modelo MMA 7361 cujas dimensões são $27,9 \mathrm{~mm}$ por $18,6 \mathrm{~mm}$; possuindo banda de frequência média de $400 \mathrm{~Hz}$ no eixo $\mathrm{X}$ e massa de $2,5 \mathrm{~g}$. O sensor foi fixado na tampa dianteira do motor abaixo do eixo do mesmo. O eixo de coleta do acelerômetro foi o eixo X, ilustrado pela Fig. 2 e a montagem em bancada está ilustrada na Fig. 3.

Para a coleta dos dados, o acelerômetro foi controlado por um microcontrolador Arduino MEGA 2560. Após a coleta de dados, os mesmos foram salvos em arquivo de planilhas para processamentos posteriores por meio do software MatLab®.

Os detalhes dos rolamentos utilizados e suas falhas são mostrados na Fig. 4. Ao todo foram realizadas 20 coletas com frequência de amostragem em $200 \mathrm{~Hz}$, cada coleta com 2 minutos de duração, gerando, para cada coleta, um total de 24.000 amostras.

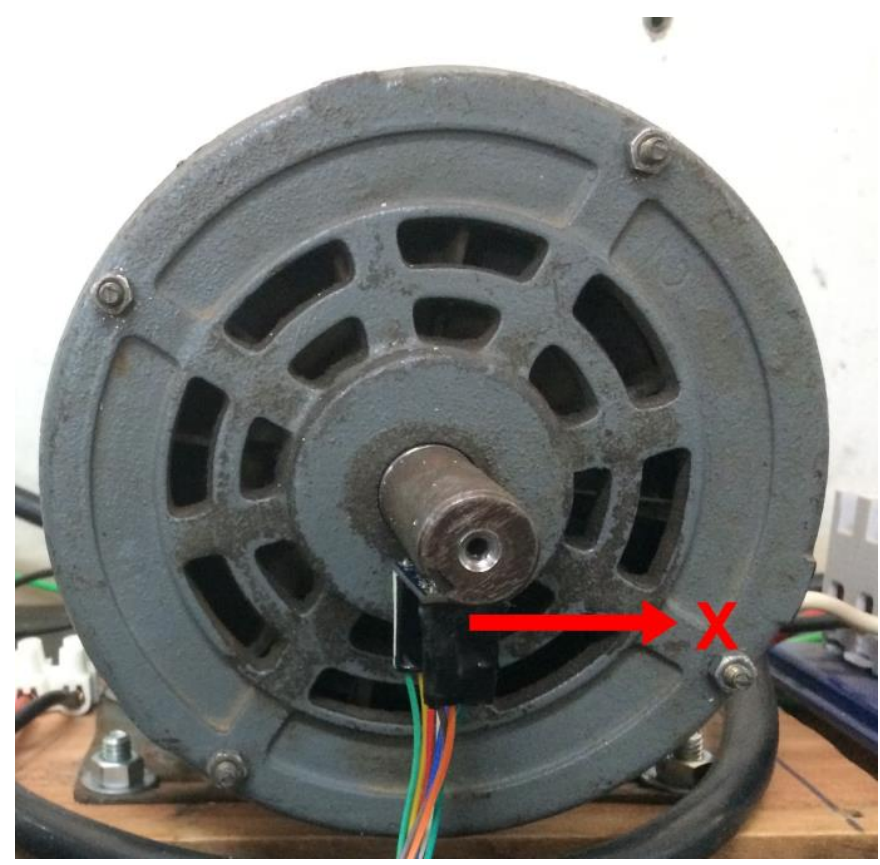

Fig. 2 Acoplamento do acelerômetro no motor e representação do eixo coletado.

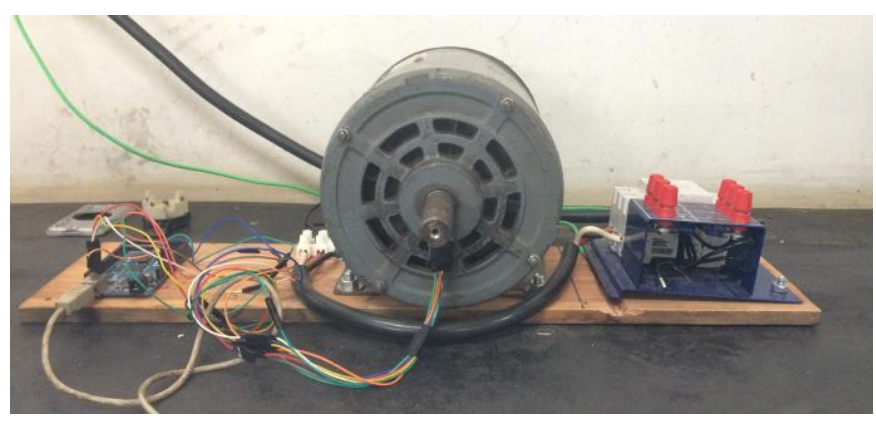

Fig. 3 Montagem do experimento em bancada. 


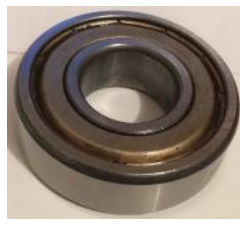

(a)

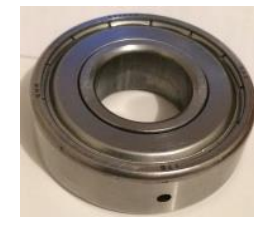

(b)

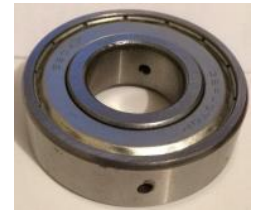

(c)
Fig. 4 Rolamentos utilizados no experimento. (a) Rolamento sem falhas, (b) Rolamento com falha em pista externa e (c) Rolamento com falha nas duas pistas.

\subsection{Extração de Parâmetros}

Para a extração de parâmetros foram utilizadas as EOS (Mendel, 1991). O uso de tal método para extração de parâmetros se deu devido o experimento ser executado em uma fonte de ruídos como um motor de indução, tal método se apresentou como alternativa robusta aos ruídos de fundo. Além disso, as EOS possuem boa capacidade de representação de sinais de vibração (Barbosa et. al., 2016). Foram utilizadas as aproximações dos cumulantes de segunda e quarta ordens para vetores de comprimento finito. As formulações encontram-se nas equações (1) e (2).

$\hat{C}_{2, x}:=\frac{2}{N} \sum_{n=0}^{N} x[n] x[\bmod [n, N]]$

$\hat{C}_{4, x}:=\frac{2}{N} \sum_{n=0}^{N} x[n] x^{3}[\bmod [n, N]]-$ $\frac{2}{N^{2}} \sum_{n=0}^{N} x[n] x[\bmod [n, N]] \sum_{n=0}^{N} x^{2}[n]$

Em que $N$ é o comprimento do vetor de entrada $x$, e $\bmod [n, N]$ é o resto da divisão da posição $n$ por $N$.

\subsection{Projeto do Classificador}

\subsubsection{Curvas Principais}

Para o projeto do classificador foram utilizadas Curvas Principais. Curvas Principais são uma generalização nãolinear da análise de componentes principais e têm sido utilizadas em problemas de agrupamento e classificação com resultados promissores, como visto em Moraes e Ferreira (2016). Desenvolvido por Hastie e Stuetzle (1989), as CP são uma técnica não paramétrica e sua forma é sugerida pelos dados que nela se projetam.

Após seu primeiro desenvolvimento, outros algoritmos para extrair CP foram surgindo com o objetivo de melhorar seu desempenho computacional, além de garantir a convergência, dado que o primeiro algoritmo apresentava elevada atração a mínimos locais, reduzindo o desempenho do método. Dentre os algoritmos, encontra-se o k-segmentos, proposto por Verbeek, Vlasis e Krose (2001). Este algoritmo obtém uma CP de maneira incremental, por segmentos de reta, possui menor suscetibilidade a mínimos locais e convergência prática garantida. $\mathrm{O}$ algoritmo k-segmentos é dividido em 3 passos.
Passo 0: Obtém-se o primeiro segmento, este utilizando como agrupamento todo o conjunto de dados. O segmento é obtido na direção da primeira componente principal e possui comprimento de $3 / 2$ do desvio padrão dos dados.

Passo 1: Um segundo segmento é inserido em um novo agrupamento realizado por meio do algoritmo $k$-means baseando-se nas regiões de Voronoi. Após a obtenção do segundo segmento, o primeiro segmento é recalculado devido à alteração em seu agrupamento. Para os demais segmentos ocorre o mesmo, inserção de novo segmento e recálculo dos segmentos cujo agrupamento houve mudança.

Passo 2: Passo de teste de convergência do algoritmo, para isto, verifica-se se o número de segmentos $k$ atingiu o valor de segmentos máximo estipulado pelo usuário $\left(K_{\max }\right)$ ou se o maior agrupamento possível tem menos de 3 segmentos. Caso não sejam atendidas tais condições, o algoritmo retorna ao passo 1 .

A Fig. 5 ilustra um fluxograma do algoritmo k-segmentos para obtenção das CP.

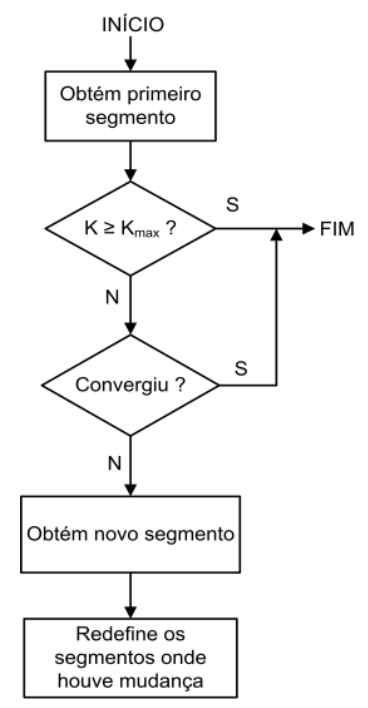

Fig. 5 Fluxograma do algoritmo k-segmentos para obtenção de CP.

\subsubsection{Curvas Principais Como Classificador}

Dentro das abordagens de classificação, a abordagem utilizada neste trabalho foi a supervisionada, abordagem em que se conhece os rótulos de cada classe (Theodoridis e Koutroumbas, 2003). Baseando-se nesta abordagem, foi projetado o classificador baseado nas $\mathrm{CP}$.

Para excutar a tarefa de classificar os padrões obtidos pelos parâmetros extraídos dos cumulantes, foi projetada, dentro do conjunto de desenvolvimento, uma $\mathrm{CP}$ para cada padrão de sinal do motor, utilizando os dados de cada padrão dentro do conjunto de desenvolvimento.

Após a extração das $\mathrm{CP}$, foi realizado o mapeamento das distâncias euclidianas de cada evento para as 3 curvas obtidas, logo, foram calculadas 3 distâncias para cada evento. A regra de classificação se deu atribuindo cada evento à 
curva cuja menor distância foi calculada. Ou seja, o evento é atribuído ao padrão ao qual se tem menor distância da curva daquele padrão, por exemplo, se um determinado evento tem menor distância à curva relativa ao padrão do motor sem falha, este é classificado como evento referente ao motor sem falhas no rolamento.

\section{RESULTADOS E DISCUSSÕES}

Cada sinal obtido contém 24.000 amostras, estas foram divididas em eventos contendo, cada um, 1.500 amostras. Sinais da aceleração normalizados em função do número de amostras são apresentados na Fig. 6.

Após a aquisição de sinais foram extraídos os cumulantes de segunda e quarta ordens com atraso zero. A Fig. 7 mostra o espaço gerado pelos cumulantes. Observe que a dimensão do problema foi reduzida de 1.500 para apenas 2. Em sequência, foram geradas as CP. Para este problema, foram abordadas três topologias de CP: com 5, 10 e 15 segmentos. Para cada topologia, foi executado o modelo 100 vezes variando aleatoriamente, em cada execução, o conjunto de treino e teste e tomadas como métricas de desempenho do classificador a média e o desvio-padrão das taxas de acerto do mesmo. Em seguida, foi variado o tamanho do conjunto de desenvolvimento da curva e tomadas, novamente, as 100 execuções variando aleatoriamente os conjuntos de treino e teste. O tamanho do conjunto de desenvolvimento da $\mathrm{CP}$ foi variado de 50 a 250 em intervalos de 50. Tal comparativo tem o propósito de avaliar o modelo de Curva Principal com bom desempenho e menor custo computacional, além de eventual caso de overtraining. Os resultados de taxas de acertos do classificador para os modelos com 5, 10 e 15 segmentos para diferentes tamanhos de conjunto de dados $N_{t}$ estão contidos, respectivamente, nas Tabelas 1, 2 e 3 .

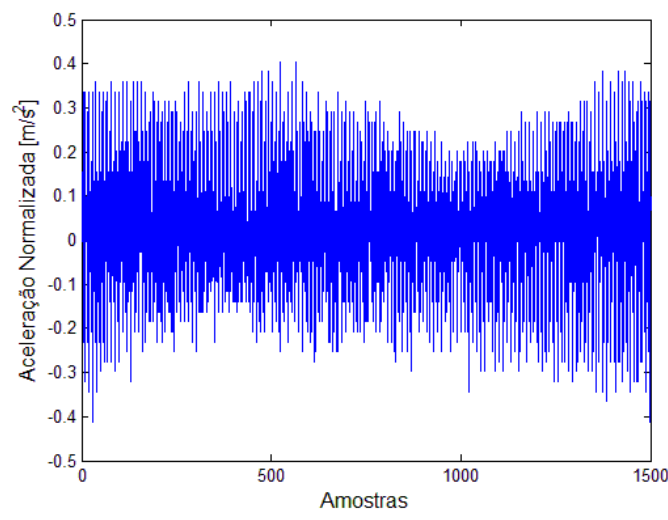

(a)

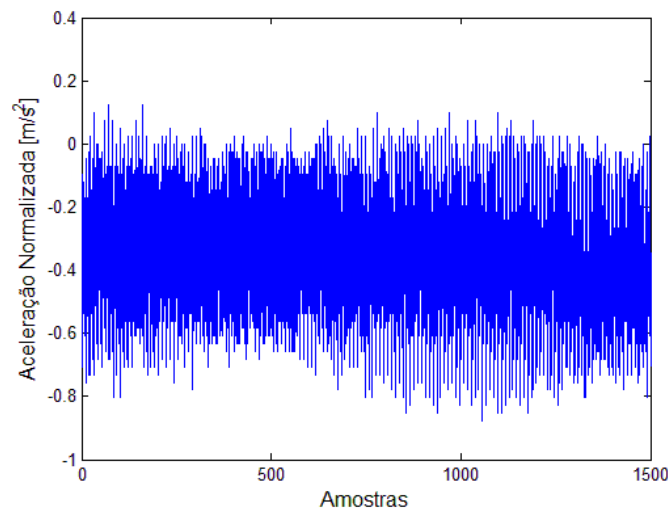

(b)

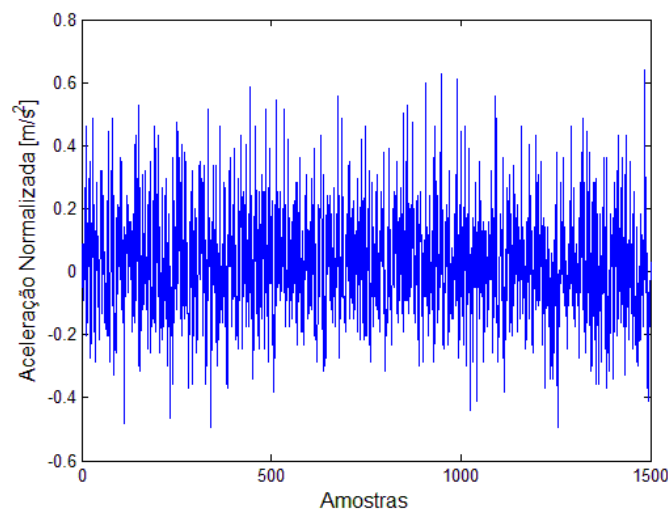

(c)

Fig. 6 Sinais coletados do motor nas 3 condições analisadas: (a) motor sem falha, (b) motor com falha na pista externa e (c) motor com falha nas duas pistas. 


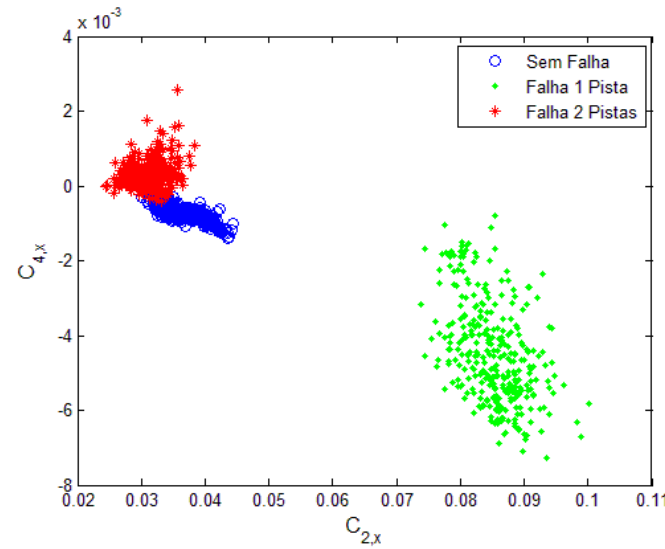

Fig 7. Espaço de parâmetros obtido pelos cumulantes contendo todo o conjunto de dados.

Tabela 1. Taxas de acerto [\%] para topologia de 05 $\operatorname{segmentos}(\mu \pm \sigma)$

\begin{tabular}{|c|c|c|c|c|c|c|}
\hline \multirow{2}{*}{$N_{\mathrm{t}}$} & \multicolumn{2}{|c|}{$\begin{array}{c}\text { Classe Sem } \\
\text { Falha }\end{array}$} & \multicolumn{2}{c|}{$\begin{array}{c}\text { Classe Falha 1 } \\
\text { Pista }\end{array}$} & \multicolumn{2}{c|}{$\begin{array}{c}\text { Classe Falha 2 } \\
\text { Pistas }\end{array}$} \\
\cline { 2 - 7 } & Treino & Teste & Treino & Teste & Treino & Teste \\
\hline \multirow{2}{*}{50} & 98,68 & 98,02 & 100,00 & 100,00 & 97,76 & 96,76 \\
& $\pm 1,46$ & $\pm 1,71$ & $\pm 0,00$ & $\pm 0,00$ & $\pm 1,80$ & $\pm 1,49$ \\
\hline \multirow{2}{*}{100} & 98,55 & 98,22 & 100,00 & 100,00 & 97,93 & 97,67 \\
& $\pm 1,37$ & $\pm 1,37$ & $\pm 0,00$ & $\pm 0,00$ & $\pm 1,24$ & $\pm 1,19$ \\
\hline \multirow{2}{*}{150} & 98,77 & 98,74 & 100,00 & 100,00 & 97,74 & 97,70 \\
& $\pm 0,94$ & $\pm 0,91$ & $\pm 0,00$ & $\pm 0,00$ & $\pm 0,95$ & $\pm 0,94$ \\
\hline \multirow{2}{*}{200} & 98,90 & 98,93 & 100,00 & 100,00 & 97,58 & 97,61 \\
& $\pm 0,54$ & $\pm 0,84$ & $\pm 0,00$ & $\pm 0,00$ & $\pm 0,79$ & $\pm 1,26$ \\
\hline \multirow{2}{*}{250} & 98,91 & 98,77 & 100,00 & 100,00 & 97,38 & 97,26 \\
& $\pm 0,44$ & $\pm 1,15$ & $\pm 0,00$ & $\pm 0,00$ & $\pm 0,78$ & $\pm 1,81$ \\
\hline
\end{tabular}

Tabela 2. Taxas de acerto [\%] para topologia de 10 segmentos $(\mu \pm \sigma)$

\begin{tabular}{|c|c|c|c|c|c|c|}
\hline \multirow{2}{*}{$N_{t}$} & \multicolumn{2}{|c|}{$\begin{array}{c}\text { Classe Sem } \\
\text { Falha }\end{array}$} & \multicolumn{2}{|c|}{$\begin{array}{c}\text { Classe Falha } 1 \\
\text { Pista }\end{array}$} & \multicolumn{2}{|c|}{$\begin{array}{c}\text { Classe Falha } 2 \\
\text { Pistas }\end{array}$} \\
\hline & Treino & Teste & Treino & Teste & Treino & Teste \\
\hline 50 & $\begin{array}{l}99,10 \\
\pm 1,22\end{array}$ & $\begin{array}{l}98,40 \\
\pm 1,26\end{array}$ & $\begin{array}{c}100,00 \\
\pm 0,00\end{array}$ & $\begin{array}{c}100,00 \\
\pm 0,00\end{array}$ & $\begin{array}{l}98,00 \\
\pm 1,84\end{array}$ & $\begin{array}{l}96,51 \\
\pm 1,53\end{array}$ \\
\hline 100 & $\begin{array}{l}99,03 \\
\pm 0,92\end{array}$ & $\begin{array}{l}98,71 \\
\pm 0,83\end{array}$ & $\begin{array}{c}100,00 \\
\pm 0,00\end{array}$ & $\begin{array}{c}100,00 \\
\pm 0,00\end{array}$ & $\begin{array}{l}98,28 \\
\pm 1,39\end{array}$ & $\begin{array}{l}97,90 \\
\pm 1,04\end{array}$ \\
\hline 150 & $\begin{array}{l}98,93 \\
\pm 0,84\end{array}$ & $\begin{array}{l}98,87 \\
\pm 0,85\end{array}$ & $\begin{array}{c}100,00 \\
\pm 0,00\end{array}$ & $\begin{array}{c}100,00 \\
\pm 0,00\end{array}$ & $\begin{array}{c}98,31 \\
1,20\end{array}$ & $\begin{array}{c}98,31 \\
1,08\end{array}$ \\
\hline 200 & $\begin{array}{l}98,99 \\
\pm 0,65\end{array}$ & $\begin{array}{l}99,12 \\
\pm 0,81\end{array}$ & $\begin{array}{l}100,00 \\
\pm 0,00\end{array}$ & $\begin{array}{l}100,00 \\
\pm 0,00\end{array}$ & $\begin{array}{l}98,41 \\
\pm 0,74\end{array}$ & $\begin{array}{l}98,41 \\
\pm 1,06\end{array}$ \\
\hline 250 & $\begin{array}{l}98,96 \\
\pm 0,66\end{array}$ & $\begin{array}{l}98,91 \\
\pm 1,45\end{array}$ & $\begin{array}{l}100,00 \\
\pm 0,00\end{array}$ & $\begin{array}{l}100,00 \\
\pm 0,00\end{array}$ & $\begin{array}{l}98,50 \\
\pm 0,62\end{array}$ & $\begin{array}{l}98,50 \\
\pm 1,52\end{array}$ \\
\hline
\end{tabular}

Tabela 3. Taxas de acerto [\%] para topologia de 15 $\operatorname{segmentos}(\mu \pm \sigma)$

\begin{tabular}{|c|c|c|c|c|c|c|}
\hline \multirow{2}{*}{$\mathrm{N}_{\mathrm{t}}$} & \multicolumn{2}{|c|}{$\begin{array}{c}\text { Classe Sem } \\
\text { Falha }\end{array}$} & $\begin{array}{c}\text { Classe Falha 1 } \\
\text { Pista }\end{array}$ & \multicolumn{2}{c|}{$\begin{array}{c}\text { Classe Falha 2 } \\
\text { Pistas }\end{array}$} \\
\cline { 2 - 7 } & Treino & Teste & Treino & Teste & Treino & Teste \\
\hline \multirow{2}{*}{50} & 99,20 & 98,27 \\
& $\pm 1,10$ & $\pm 1,08$ & $\begin{array}{c}100,00 \\
\pm 0,00\end{array}$ & $\begin{array}{c}100,00 \\
\pm 0,00\end{array}$ & $\begin{array}{c}98,04 \\
\pm 1,71\end{array}$ & $\begin{array}{c}96,46 \\
\pm 1,68\end{array}$ \\
\hline \multirow{2}{*}{100} & 99,06 & 98,87 & 100,00 & 100,00 & 98,14 & 97,40 \\
& $\pm 1,08$ & $\pm 0,80$ & $\pm 0,00$ & $\pm 0,00$ & $\pm 1,18$ & $\pm 1,18$ \\
\hline \multirow{2}{*}{150} & 99,04 & 99,05 & 100,00 & 100,00 \\
$\pm 0,80$ & $\pm 0,79$ & $\pm 0,00$ & $\begin{array}{c}98,00 \\
\pm 1,32\end{array}$ & $\begin{array}{c}98,04 \\
\pm 1,33\end{array}$ \\
\hline \multirow{2}{*}{200} & $\begin{array}{c}98,94 \\
\pm 0,67\end{array}$ & $\begin{array}{c}98,97 \\
\pm 0,92\end{array}$ & $\begin{array}{c}100,00 \\
\pm 0,00\end{array}$ & $\begin{array}{c}100,00 \\
\pm 0,00\end{array}$ & $\begin{array}{c}98,43 \\
\pm 1,15\end{array}$ & $\begin{array}{c}98,46 \\
\pm 1,29\end{array}$ \\
\hline \multirow{2}{*}{250} & 98,89 & 98,90 & 100,00 & 100,00 & 98,76 & 98,77 \\
$\pm 0,53$ & $\pm 1,13$ & $\pm 0,00$ & $\pm 0,00$ & $\pm 0,90$ & $\pm 1,38$ \\
\hline
\end{tabular}

Analisando a Fig. 7 e as Tabelas 1, 2 e 3, pode-se observar os seguintes pontos importantes: (i) os resultados de treino e teste para a Classe de Falha em 1 pista obtiveram taxas de acerto de $100 \%$ dada a separação linear desta classe para as demais; (ii) apesar de as classes Sem Falha e Falha 2 pistas não serem linearmente separáveis no espaço de parâmetros obtido (Fig. 7), resultados acima de 96\% foram alcançados; (iii) vale destacar que altas taxas de acerto foram obtidas mesmo para pequenos conjuntos de treinamento. Tal resultado possui importância em aplicações reais, dado que nestes casos, geralmente, dispõem-se de pequenos conjuntos de dados de treinamento.

Em todas as topologias, pode-se constatar também que o aumento do conjunto de treinamento não implicou necessariamente em aumento das taxas de acerto médias ou redução dos desvios-padrão para todas as classes. Tal situação também é válida para o aumento da complexidade das CP, em que o aumento da complexidade não implicou em melhoria de desempenho. Fazendo uma análise comparativa dentre os tamanhos dos conjuntos de treinamento e as topologias, o melhor desempenho por análise de maior taxa de acerto, menor desvio e prioridade ao teste, foi a topologia de 10 segmentos com conjunto de dados de treino $N_{t}=200$. Contudo, tal escolha não descartaria o uso de uma topologia de menor complexidade, como a topologia de 5 segmentos que obteve resultados próximos com menor custo computacional.

\section{CONCLUSÃO}

Por meio de análises realizadas durante o projeto do classificador e a análise dos resultados, foram obtidos resultados promissores para o método proposto dado que, em arquiteturas simples, taxas de acerto acima de $95 \%$ foram obtidas com baixos conjuntos de treinamento, mesmo para 
classes não linearmente separáveis, como o caso das classes sem falha e classe falha 2 pistas.

Vale ressaltar, que o método desenvolvido apresenta reduzida complexidade computacional na fase operacional, visto que para se classificar um evento, basta calcular a distância Euclidiana do mesmo às três $\mathrm{CP}$ que representam as classes consideradas, já que a construção das $\mathrm{CP}$ é realizada off-line.

Para trabalhos futuros visa-se uma maior análise de falhas, com mais variedade e combinações das mesmas e uma possível implementação online em sistema embarcado. Podendo assim, propiciar um método de classificação de falhas eficiente e de baixos custos de operação, garantindo uma produção de maior qualidade e segurança nas indústrias.

\section{AGRADECIMENTOS}

Agradecimentos pelo aporte financeiro à pró-reitoria de pesquisa da Universidade Federal de Lavras (PRP -UFLA) CAPES, FAPEMIG e CNPq e à Bobinadora Lavrense pelo empréstimo do motor utilizado nos experimentos.

\section{REFERÊNCIAS}

Barbosa, T. S. et al. Fault Detection and Classification in Cantilever Beams Through Vibration Signal Analysis and Higher-Order Statistics. Journal of Control, Automation and Electrical Systems, v. 27, n. 5, p. 535-541, 2016.

Culbert, I.; Letal, J. Signature Analysis for Online Motor Diagnostics: Early Detection of Rotating Machine Problems Prior to Failure. IEEE Industry Applications Magazine, v. 23, n. 4, p. 76-81, 2017.

Darong, Huang et al. A new incipient fault diagnosis method combining improved RLS and LMD algorithm for rolling bearings with strong background noise. IEEE Access, v. 6, p. 26001-26010, 2018.

Gongora, W. S.; Goedtel, A.; da Silva, S. A. O.; Graciola, C. L.. Neural Approach to Fault Detection in Three-phase Induction Motors. IEEE Latin America Transactions, v. 14, n. 3, p. 1279-1288, 2016.

Hastie, T. J., Stuetzle, W. Principal Curves, Journal of the American Statistical Association, v. 84, n. 406, pp. 502-516, 1989.

Liao, W; Wang, Y. Data-driven Machinery Prognostics Approach using in a Predictive Maintenance Model. JCP, v. 8, n. 1, p. 225-231, 2013.

Mendel, J. M. Tutorial on higher-order statistics (spectra) in signal processing and system theory: Theoretical results and some applications. Proceedings of the IEEE, v. 79, n. 3, p. 278-305, 1991.

Moraes, E. C. C.; Ferreira, D. D., A principal curve-based method for data clustering. In: 2016 International Joint Conference on Neural Networks (IJCNN), 2016, Vancouver. 2016 International Joint Conference on Neural Networks (IJCNN). p. 3966-3971.

Park, Y.; Jeong, M.; Lee, S. B.; Antonino-Daviu, J. A.; Teska, M. Influence of Blade Pass Frequency Vibrations on MCSA-Based Rotor Fault Detection of Induction Motors. IEEE Transactions on Industry Applications, v. 53, n. 3, p. 2049-2058, 2017.

Thoben, K; Wiesner, S; Wuest, T. "Industrie 4.0" and Smart ManufacturingA Review of Research Issues and Application Examples. Int. J. of Automation Technology Vol, v. 11, n. 1, 2017.

Tian, Jing et al. Motor bearing fault detection using spectral kurtosis-based feature extraction coupled withk-nearest neighbor distance analysis. IEEE Transactions on Industrial Electronics, v. 63, n. 3, p. 1793-1803, 2016.

Verbeek, J. J.; Vlassis, N.; Krose, B. A soft k-segments algorithm for principal curves, Proceedings of International Conference on Artificial Neural Networks, pp. 450-456, 2001

Theodoridis, S.; Koutroumbas K. Pattern recognition. 2 ed. Academic Press, 2003. 689p.

$\mathrm{Yu}$, Xiu-lian et al. Fault detection method for the rolling bearings of metro vehicle based on RBF neural network and wavelet packet transform. In: 2013 IEEE International Conference on Intelligent Rail Transportation Proceedings. IEEE, 2013. p. 245-248. 